\title{
CRYPTREC Project \\ - Cryptographic Evaluation Project for the Japanese Electronic Government -
}

\author{
Hideki Imai ${ }^{1}$ and Atsuhiro Yamagishi ${ }^{2}$ \\ 1 Institute of Industrial Science, The University of Tokyo, \\ Roppongi, Minato-ku, Tokyo 106-8558, Japan \\ imai@iis.u-tokyo.ac.jp \\ 2 Information-Technology Promotion Agency,Japan \\ Bunkyo Green Court Center Office \\ 2-28-8 Honkomagome, Bunkyo-ku, Tokyo 113-6591, Japan \\ a-yamagi@ipa.go.jp
}

\begin{abstract}
We will describe the outline of the cryptographic technology evaluation project in Japan and those present conditions. The purpose of this project is that the cyptographic technology which the Japanese Government uses is evaluated and listed. Selected cryptographic technology will be used in the information security system which the Japanese Government will use in the future.

Keywords. Cryptographic technology, Symmetric ciphers, Asymmmetric ciphers,Evaluation
\end{abstract}

\section{Background}

Creating the common security basis is one of the most important tasks for the Japanese electronic government of which the infrastructure and primary systems will be constructed by FY 2003. Cryptographic techniques are particularly important and indispensable components of the electronic government because these not only provide information confidentiality and prevent information falsification, but also assure electronic authentication. Because of this importance, it has been pointed out domestically that the Japanese national government should adopt a cryptography usage policy in order to ensure that cryptography is integrated properly into the electronic government. Internationally, on the other hand, the ISO/IEC JTC1 has begun efforts aimed at standardizing cryptographic algorithms. CRYPTREC Project is an essential part of the MITI Action Plan for a Secure E- Government, which was announced by the Ministry of International Trade and Industry (MITI) in April 2000. MITI has entrusted the IPA with the implementation of this project.

\section{Purposes and Project Implementation}

The purpose of this project is to publish a technical report by the end of March 2001. It is to include a list of characteristics on cryptographic techniques that 
will be proposed through a call for submission applicable to the Japanese electronic government. In order to make such a list, the action plan of the project contains investigation and evaluation of the proposed cryptographic techniques in terms of security, implementation and other characteristics from the objective viewpoints of various specialists. Four governmental offices (the Management and Coordination Agency, the Ministry of International Trade and Industry, the Ministry of Posts and Telecommunications and the Defense Agency, Japan) jointly organized CRYPTREC (CRYPTREC: CRYPTography Research \& Evaluation Committee) to carry out this project. The committee, which is composed of prominent cryptography specialists in Japan, will evaluate the submitted cryptographic techniques.@ Cryptographic Techniques that will be evaluated are the four types of techniques considered indispensable in the electronic government:

(1) asymmetric cryptographic schemes

(2) symmetric ciphers

(3) hash functions

(4) pseudorandom number generators.

The evaluation will be conducted in two phases: the screening test phase and the detailed evaluation phase. The latter will be carried out on the proposals that have passed the screening tests. The evaluation guidelines are to be established by the committee.

Reports, including the evaluation results, will be compiled by the committee following due and proper consideration on fairness and transparency, and will be announced on web pages hosted by the IPA.

\section{Status of Subscriptions}

CRYPTREC received 48 proposals for Call for Cryptographic Techniques. The following table shows the number of submissions in each category. At present, CRYPTREC is evaluating these candidates from the viewpoints of both security and efficiency of implementation.

Table 1. Subscriptions

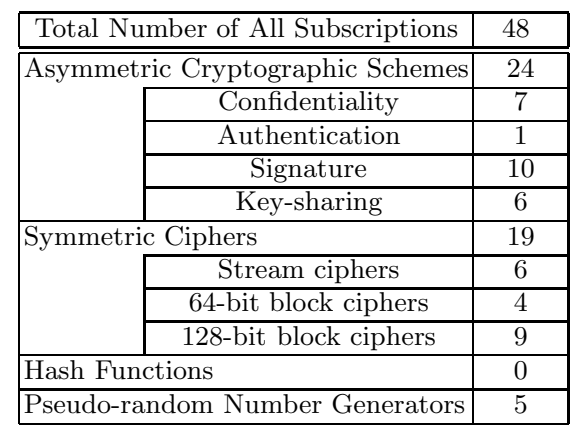

The detail of the project will be found at the following. URL:http://www.ipa.go.jp/security/enc/CRYPTREC/index-e.html 